ORIGINAL ARTICLE

\title{
Neuroradiological aspects of subdural haemorrhages
}

\author{
S Datta, N Stoodley, S Jayawant, S Renowden, A Kemp
}

Arch Dis Child 2005;90:947-951. doi: 10.1136/adc.2002.021154

See end of article for authors' affiliations

....................

Correspondence to: Dr S Datta, Specialist Registrar in Radiology, Department of Radiology, University Hospital of Wales, Heath Park, Cardiff CF14 4XW, UK; shalinidatta@btinternet. com

Accepted 15 June 2004

\begin{abstract}
Aims: To review the neuroimaging of a series of infants and young children admitted to hospital with subdural haemorrhage (SDH).

Methods: Neuroradiological investigations of 74 children under 2 years of age, from South Wales and southwest England, in whom an SDH or subdural effusion had been diagnosed between 1992 and 2001, were reviewed. Two paediatric neuroradiologists blinded to the original radiological report reviewed all the relevant images.

Results: Neuroradiological review of images identified radiological features which were highly suggestive of non-accidental head injury (NAHI). Interhemispheric haemorrhages and SDHs in multiple sites or of different densities were almost exclusively seen in NAHI. MRI was more sensitive in identifying SDHs of different signal characteristics, posterior and middle cranial fossa bleeds, and parenchymal changes in the brain. CT scans, if performed with suboptimal protocols, were likely to miss small subdural bleeds.

Conclusions: Guidelines for neuroimaging in suspected NAHI are recommended. A radiologist with experience in NAHI should report or review these scans. The initial investigation should be CT, but MRI will also be necessary in most cases. Head CT should be an integral part of the skeletal survey in all infants less than 6 months of age referred for child protection investigation, and in children less than 2 years where child abuse is suspected and there are neurological signs, retinal haemorrhages, or fractures.
\end{abstract}

S ubdural haemorrhage (SDH) in infants and young children has an incidence of 20-25 per 100000 in children less than 1 year, ${ }^{1-3}$ and most cases are due to physical abuse. Other causes include accidental trauma, meningitis, coagulopathy, vascular malformations, and rare metabolic disorders.

These infants present with varied and usually non-specific symptoms. Initial neuroimaging may be undertaken by radiologists without particular expertise in paediatric neuroimaging, but the initial investigations are critical to the crucial decisions which are made within the child protection and legal process. It is therefore essential that the methods of investigation and interpretation are standardised and well understood. The Royal College of Radiologists, ${ }^{4}$ the American Academy of Pediatrics, ${ }^{5}{ }^{6}$ and recently Jaspan and colleagues ${ }^{7}$ have suggested guidance in the neuroimaging of NAHI.

The diagnosis of SDH is made by cranial imaging, mainly computed tomography (CT) and magnetic resonance imaging (MRI); the features of SDH due to non-accidental head injury (NAHI) are well described.$^{8-11}$ MRI is used as an adjunct to CT in the radiological assessment of SDH to detect subdural blood in areas not well seen on $\mathrm{CT}_{1}{ }^{12-14}$ or small volume SDHs, to differentiate subdural and subarachnoid blood and to show parenchymal injuries.

Our study aims to review the neuroimaging of a series of infants and young children admitted to hospital with SDH, and highlight features typical of NAHI. We make recommendations for neuroimaging in suspected cases of abuse in young children based on these findings.

\section{SUBJECTS AND METHODS}

We undertook a retrospective analysis of 74 patients under the age of 2 years with SDH or subdural effusion diagnosed between 1992 and 2001. The following selection criteria were used to identify NAHI:

- Confession by the perpetrator

- A criminal conviction for child abuse where there was supportive evidence from unexplained extracranial injuries
- SDH and associated findings, where NAHI was concluded at case conference.

Cases from South Wales and southwest England were identified using the Welsh Paediatric Surveillance System, ICD patient coding, radiology databases, admission data, ascertainment from clinicians, pathologists, HM coroners, and expert witnesses in child protection cases.

Case notes were obtained and reviewed. Note was made of clinical features and type and timing of the imaging study. Two paediatric neuroradiologists blinded to the initial report reviewed all the images. Location of bleeding was recorded as convexity, interhemispheric, posterior fossa, middle cranial fossa, or subarachnoid space. Brain parenchymal changes were recorded as contusions, haematomas, infarcts, focal atrophy, diffuse axonal injury (DAI), focal or generalised swelling, and oedema.

MREC approval was granted.

\section{RESULTS}

Neuroradiological review was completed in all 74 cases. Forty nine cases were identified as NAHI, classified as follows:

- Confession by the perpetrator $(\mathrm{n}=12)$

- Criminal conviction $(\mathrm{n}=8)$

- NAHI agreed at case conference $(n=29)$.

NAHI was suspected in a further 11 cases, but the cases did not fulfil the study child abuse inclusion categories; these cases were excluded from the detailed radiological analysis. Three cases followed serious, independently witnessed accidents.

Abbreviations: $\mathrm{CT}$, computed tomography; DAl, diffuse axonal injury; $\mathrm{MRI}$, magnetic resonance imaging; NAHI, non-accidental head injury; $\mathrm{SDH}$, subdural haemorrhage 
Eleven SDH resulted from miscellaneous causes:

- 7 meningitis

- 2 postoperative rebleeds

- 1 haemorrhagic disease of the newborn

- 1 benign effusion which resolved on follow up.

Seventy nine per cent of NAHI cases (39/49) were less than 6 months old. Presenting symptoms varied from drowsiness and malaise to fitting and coma. One child died soon after arrival. Forty seven patients had one or more extracranial injuries such as retinal haemorrhages, bruising, or fractures. Retinal haemorrhages were common; bilateral in 25 and unilateral in 10 cases. Bruising was present in 26 children, 20 cases involving the scalp or face. Two patients had no extracranial injuries; both were under 6 months of age. Twelve infants had increased head circumference on admission. Results of lumbar puncture were documented in 19 patients. In all cases the cerebrospinal fluid was haemorrhagic.

Fractures were seen in 29/49 cases. Rib fractures were commonest (14 patients), with limb fractures in 11 and multiple fractures in 21 patients. Skull fractures were found in 11 patients; 4 had more than one fracture. Evidence of impact to the head was seen in 16 cases (11 skull fractures with or without scalp swelling and 5 with swelling alone) (table 1). In 15 of 16 children with evidence of impact, SDHs were present in multiple sites, both at and away from the impact site. Vertebral fractures were seen in one child.

In 73/74 patients CT was the first neuroimaging investigation; one had an MRI scan. Twenty five patients had MRI scans 1-12 days later. The initial CT scan was performed at a district hospital in $90 \%$ of cases. In 10 patients only $10 \mathrm{~mm}$ slices were obtained through the whole brain (eight of these children were scanned between the years 1992 and 1995), and in one patient only a post-contrast study was performed. In 7/49 children with NAHI the original CT was reported as normal or not suggestive of NAHI. The diagnosis of NAHI was subsequently made following neuroradiological review prompted by high clinical suspicion of abuse.
In seven cases, subdural collections followed meningitis (all Streptococcus pneumoniae). In six cases these were detected within 2-5 days of symptom onset, but in one case they were detected three weeks later. Two of these children had unilateral, and five bilateral collections. Subdural taps occurred in five of these seven cases, and in four of these the fluid was haemorrhagic. The initial lumbar puncture was also haemorrhagic in six of these seven cases. Bleeding followed a subdural tap in only one case. All post-meningitis subdurals were convexity collections.

Neuroradiological findings are summarised in table 2. CT and MRI were performed in 25 children, and additional SDHs were identified on MRI in 19 cases:

- 7 convexity

- 3 interhemispheric

- 5 posterior fossa

- 4 middle fossa bleeds.

Subdural collections of different appearances (density or intensity) were seen in 26 children with NAHI, and in six this feature was seen only on the MRI scan. Ultrasound was performed before CT in 12 cases; SDHs were not seen in seven of these.

Subdural collections were seen in more than two sites in $32 / 49$ in the NAHI group; in 26/49 the SDHs had components of different CT densities suggestive of bleeds of different ages. In the miscellaneous group multifocal subdural collections were only seen in the infant with coagulopathy and the two children involved in road traffic accidents (one of whom had retinal haemorrhages). The child who fell $7.5 \mathrm{ft}$ down the stairs had an isolated SDH at the site of skull fracture and no retinal haemorrhage. In all these four children, the SDHs were hyperdense on CT, consistent with acute blood.

Radiological DAI was seen in one case of NAHI. Cerebral oedema (focal or generalised) was seen in 12/49 cases. Generalised oedema associated with SDH was seen only in the NAHI group. No contusional tears were seen on CT or MRI, although in seven cases there were focal parenchymal

Table 1 Details of injury in cases with definite evidence of impact

\begin{tabular}{|c|c|c|c|}
\hline & \multicolumn{3}{|l|}{ Evidence of impact } \\
\hline & Skull fractures & Bruising & Subdural site \\
\hline Case 1 & Right parietal, left occipital & Bruising on opposite side of fracture & $\begin{array}{l}\text { Multiple SDHs of different densities, below and away from } \\
\text { fracture }\end{array}$ \\
\hline Case 2 & 2 separate parieto-occipital & Bruises to face & $\begin{array}{l}\text { Multiple SDHs of different densities, below and away from } \\
\text { fracture }\end{array}$ \\
\hline Case 3 & Left parietal & No bruising & $\begin{array}{l}\text { Multiple SDHs of different densities, below and away from } \\
\text { fracture }\end{array}$ \\
\hline Case 4 & Right parietal & Swelling at site of fracture & SDH underlying fracture \\
\hline Case 5 & Occipital & No bruising & Multiple SDHs of different densities, away from fracture \\
\hline Case 6 & Right parietal, left parietal & Swelling at site of fracture & Multiple SDHs, below and away from fracture \\
\hline Case 7 & Right parietal & Swelling at site of fracture & $\begin{array}{l}\text { Multiple SDHs of different densities, below and away from } \\
\text { fracture }\end{array}$ \\
\hline Case 8 & Left parietal linear, old occipital & Overlying cephalohaematoma & Multiple SDHs, below and away from fracture \\
\hline Case 9 & Left sided parietal & $\begin{array}{l}\text { Soft tissue swelling away from site } \\
\text { of fracture }\end{array}$ & Multiple SDHs, below and away from fracture \\
\hline Case 10 & Posterior parietal & Bruises on neck & $\begin{array}{l}\text { Multiple SDHs of different densities, below and away from } \\
\text { fracture }\end{array}$ \\
\hline Case 11 & Left posterior parietal & $\begin{array}{l}\text { Bruising on face, upper and lower arm, } \\
\text { torn frenulum, old scar on forehead }\end{array}$ & Multiple SDHs, below and away from fracture \\
\hline Case 12 & None & Bruise right side of forehead & Multiple SDHs, below and away from bruise \\
\hline Case 13 & None & Bruise right side of forehead & Multiple SDHs, below and away from bruise \\
\hline Case 14 & None & Bruised forehead & $\begin{array}{l}\text { Multiple SDHs of different densities, below and away from } \\
\text { bruise }\end{array}$ \\
\hline Case 15 & None & Right occipital bruise & $\begin{array}{l}\text { Multiple SDHs of different densities, below and away from } \\
\text { bruise }\end{array}$ \\
\hline Case 16 & None & Left parietal bruise & Multiple SDHs, below and away from bruise \\
\hline
\end{tabular}


Table 2 Imaging features in various groups

\begin{tabular}{|c|c|c|c|}
\hline & $\begin{array}{l}\text { NAHI } \\
(n=49)\end{array}$ & $\begin{array}{l}\text { Accidental trauma } \\
(n=3)\end{array}$ & $\begin{array}{l}\text { Miscellaneous } \\
(n=11)\end{array}$ \\
\hline Age (0-6 mth) & 39 & 0 & 5 \\
\hline Age (6-24 mth) & 10 & 3 & 6 \\
\hline Convexity collection & $44(7 / 25)$ & 2 & 11 \\
\hline Interhemispheric fissure bleeds & $38(3 / 25)$ & 2 & 1 \\
\hline PCF bleeds & $14(5 / 25)$ & 2 & 1 \\
\hline MCF bleeds & $9(4 / 25)$ & 2 & - \\
\hline Focal oedema & 9 & 2 & 2 \\
\hline Generalised oedema & 3 & - & - \\
\hline Parenchymal changes & $12(5 / 25)$ & - & 3 \\
\hline Subarachnoid haemorrhage & 7 & 2 & - \\
\hline Skull fracture & $\begin{array}{l}11 \text { (SDH away } \\
\text { from \# site in 10) }\end{array}$ & $\begin{array}{l}3 \text { (SDH away } \\
\text { from \# site in 1) }\end{array}$ & - \\
\hline DAI & 1 & - & - \\
\hline SDH of apparently different ages & $26(6 / 25)$ & - & - \\
\hline SDH in multiple sites & 32 & 2 & 1 \\
\hline
\end{tabular}

Figures in parentheses represent the number of children where the imaging feature was shown only on MRI but not on CT scan.

changes such as contusions, atrophy, and infarcts. Only two of these had signs suggestive of recent impact.

Definite evidence of previous abuse was present in 20 cases of NAHI (table 3). All three children involved in accidental trauma sustained a skull fracture with SDH seen at the fracture site. In one case (involved in road traffic accident) acute subdural blood was also seen distant from the fracture site.

\section{DISCUSSION}

Imaging features in NAHI are well documented and recommendations have been made regarding imaging in NAHI. CT should be the first imaging investigation (due to its availability, the relative ease with which sick infants can be scanned, and its ability to detect acute blood). MRI should be performed as a second line investigation within the first few days following injury and for follow up. ${ }^{6{ }^{14}}$ Advanced MR techniques such as MR spectroscopy may give useful prognostic information at relatively early stages following the injury, ${ }^{15}$ and MRI diffusion weighted imaging shows patterns of abnormality consistent with hypoxic-ischaemic changes following NAHI, ${ }^{16}$ a finding which also has prognostic significance. ${ }^{118}$

Previous studies have shown improved detection of $\mathrm{SDH}$ with MRI compared to $\mathrm{CT},,^{14}$ although subarachnoid haemorrhage may be better detected by CT scans than MRI. ${ }^{19}$ Other MRI studies have also shown SDH at sites poorly seen on CT. ${ }^{12}$ Our study confirms these findings: some SDHs of low CT density, middle and posterior cranial fossa bleeds, and parenchymal changes were detected only on MRI scanning in several cases.

DAI was found in only one case, consistent with neuropathological evidence suggesting that DAI is less common following NAHI than previously thought. ${ }^{20}$ CT may underestimate DAI, which is better seen on MRI, but this was only available in half of the NAHI cases. In the neuropathological study of fatal NAHI by Geddes et al, brain weight was increased in $82 \%$ of cases, ${ }^{21}$ suggesting cerebral oedema. Our series highlights the association of generalised or focal oedema in NAHI. Although CT is less sensitive in detecting cerebral oedema or ischaemia, particularly when performed early, diffusion weighted MRI is exquisitely sensitive at showing these changes very early after injury, ${ }^{16}{ }^{17}$ and should be considered as a part of the MRI protocol in NAHI where available.

The finding of SDH in different sites or of apparently different ages or of generalised oedema in association with SDH is strongly suggestive of NAHI. The commonest site for $\mathrm{SDH}$ in our study was over the cerebral convexities with isolated convexity collections being the least discriminatory

Table 3 Evidence to support there having been repeated episodes of abuse in cases with SDHs of different ages

\begin{tabular}{ll}
\hline Case & Evidence \\
\hline Case 1 & Multiple bruises, some noted few weeks before admission, torn frenulum, old rib fracture \\
Case 2 & Old rib fracture \\
Case 3 & Previous admission with subconjunctival haemorrhage and sudden onset squint, retinal haemorrhages \\
Case 4 & Multiple bruises, cigarette burns, adult bite marks, NAl queried on previous admission, retinal haemorrhages \\
Case 5 & Multiple bruises on scalp and chest, retinal haemorrhages, seen by GP 3 times in 2 weeks prior to admission \\
Case 6 & Presented with bruises two weeks previously, retinal haemorrhages \\
Case 7 & Two week history of being unwell, shaking admitted, retinal haemorrhages \\
Case 8 & Multiple bruises, cigarette burns, retinal haemorrhages, witnessed episodes of repeated abuse \\
Case 9 & Multiple bruises, retinal haemorrhages, increasing OFC noted on previous admission \\
Case 10 & Old fractures, retinal haemorrhages \\
Case 11 & Bruising around chest, failure to thrive, retinal haemorrhages, father admitted shaking \\
Case 12 & Seen previously with episodes of apnoea and painful leg \\
Case 13 & Repeated admissions with vomiting and increasing OFC \\
Case 14 & Previous admission with rib fractures, retinal haemorrhages \\
Case 15 & Old rib fractures \\
Case 16 & Multiple bruises, bruised frenulum, retinal haemorrhages \\
Case 17 & Old rib fractures, retinal haemorrhages \\
Case 18 & Multiple bruises, concern regarding increasing OFC on previous admission \\
Case 19 & Two previous admissions with fits 1 week apart, scratches on face \\
Case 20 & Admitted previously with bruising, retinal haemorrhages \\
\hline
\end{tabular}

$\mathrm{NAl}$, non-accidental injury; OFC, occipitofrontal circumference. 


\section{What is already known on this topic}

- SDH in infants raises the possibility of NAHI

- NAHI may be missed if not thoroughly investigated

\section{What this study adds}

- Neuroimaging features highly suggestive of NAHI

- Clear guidelines for investigation in suspected NAHI feature. The interhemispheric fissure was the next commonest site, followed by the posterior and middle cranial fossa. This is similar to the findings of Sato and colleagues, ${ }^{14}$ but differs from the observations of Barlow and colleagues, ${ }^{13}$ who cite the subtemporal region as the commonest site of SDH in NAHI. Interhemispheric blood was most often seen following NAHI; indeed, apart from NAHI, SDH in the interhemispheric fissures, and posterior and middle cranial fossa was only otherwise seen following severe accidental trauma (cases of road traffic accident) and coagulopathy.

Most children with NAHI were 6 months old or less, and most had extracranial injuries and retinal haemorrhages. However, two of this group had SDH with no extracranial injuries. These two infants presented with neurological symptoms and were imaged on the basis of a discrepancy between history and clinical presentation. Sato and colleagues $^{14}$ and Morris and colleagues ${ }^{22}$ have also cited cases where SDHs were the only objective finding of abuse.

SDHs of apparently different ages were found in 26 patients with NAHI. Legal questions often arise regarding the possibility of rebleeding into pre-existing SDH in these cases. In 20/26 cases there was extracranial evidence of previous abuse, supporting the interpretation that the SDHs were due to different episodes of NAHI. These children presented with either signs of raised intracranial pressure or encephalopathy on a background of having generally being unwell. It is accepted that rebleeding can occur following minimal trauma in the elderly population. The pattern of the more acute bleeding in cases of NAHI is different to that seen in adult rebleeds, being usually multifocal rather than unifocal, of small volumes, and in a distribution typical of that seen in the primary NAHI. Therefore, this pattern of bleeding in the presence of subdural haematomas of apparently different ages is more likely to be due to further episodes of abuse rather than to spontaneous, synchronous multifocal rebleeds. Also the volumes of blood associated with these possible rebleeds is rarely of such a volume as to cause either a significant acute rise in intracranial pressure or to be the direct cause of an acute encephalopathic illness. We did not come across any cases where further SDH arose spontaneously in post-meningitic effusions. Rebleeding occurred only in one patient after surgical evacuation.

Any young child with neurological symptoms, retinal haemorrhages of unknown origin, or any other features of non-accidental injury should have a head CT scan. Based on our study findings and the high incidence of SDHs in children less than 6 months, we would recommend that a head CT scan should be part of the "skeletal survey" in children under 6 months of age where physical abuse is suspected. This condition presents with a variety of clinical signs and symptoms and carries a serious morbidity. ${ }^{1}$ We believe that if a clinician has sufficient clinical concern to request a skeletal survey, then a CT head scan should be performed to look for intracranial injury.

Suboptimal CT protocols were used at a few hospitals. Slices of only $10 \mathrm{~mm}$ throughout the whole head, or postcontrast only studies are more likely to miss small SDHs, especially those in the posterior fossa. MRI performed in the acute stage as the first neuroimaging investigation may miss small volume acute SDH and subarachnoid haemorrhages.
Ultrasound has no role in excluding an $\mathrm{SDH}$, even in experienced hands, but may be used to monitor resolution of SDH diagnosed on CT/MRI and in looking for subtle shearing lesions at grey-white interface.

The introduction of a more uniform protocol in the investigation of these cases would be of great value, and we endorse the proposals of Jaspan and colleagues ${ }^{7}$ who rightly emphasise the importance of early MRI in these cases. Optimal neuroimaging is important from the point of view of child protection, and the results have implications for the child's long term neurodevelopmental outcome. The interpretation of these scans is also of great importance, and we recommend that these cases should be reported or reviewed by a radiologist experienced in paediatric neuroimaging.

When is neuroimaging indicated?

- We recommend that it should be performed to guidelines in all young children with unexplained neurological symptoms, retinal haemorrhage, or non-accidental fractures, or if blood stained CSF is found on lumbar puncture in encephalopathic infants.

- We recommend that it should be performed to guidelines in all children under 6 months of age where sufficient grounds exist for considering the possibility of child abuse.

- A CT scan should be strongly considered as part of the skeletal survey when it forms part of the investigation of any child who is being investigated for physical abuse.

\section{Conclusions}

- MRI adds further information to CT findings in neuroimaging children with suspected NAHI and should be performed in all cases.

- Adherence to published imaging guidelines will improve quality of diagnosis.

- Interhemispheric haemorrhages, and SDHs in multiple sites or of different densities suggest NAHI. In association with history, clinical presentation, and other investigations, these findings confirm the probability that the infant has suffered serious physical abuse

- Neuroimaging should be performed in all infants under 6 months of age where the possibility of physical abuse is being investigated radiologically.

- Imaging studies should be reported or reviewed by radiologists with experience in paediatric neuroimaging.

\section{Authors' affiliations}

S Datta, A Kemp, Dept of Neuroradiology and Child Health, University Hospital of Wales, Cardiff, UK

N Stoodley, S Renowden, Dept of Neuroradiology, Frenchay Hospital, Bristol, UK

S Jayawant, Dept of Paediatrics, John Radcliffe Hospital, Oxford, UK

Competing interests: none declared

\section{REFERENCES}

1 Kemp AM, Stoodley N, Cobley C, et al. Apnoea and brain swelling in nonaccidental head injury. Arch Dis Child 2003;88:472-6.

2 Barlow KM, Minns RA. Annual incidence of shaken impact syndrome in young children. Lancet 2000;356:1571-2. 
3 Jayawant S, Rawlinson A, Gibbon F, et al. Subdural haemorrhages in infants: population based study. BMJ 1998;317:1558-61.

4 The Royal College of Radiologists. Making the best use of a department of clinical radiology: guidelines for doctors, 5th edn. London: Royal College of Radiologists, 2003.

5 American Academy of Pediatrics Committee on Child Abuse and Neglect. Shaken baby syndrome: inflicted cerebral trauma. Pediatrics 1993;92:872-5. 6 American Academy of Pediatrics. Diagnostic imaging of child abuse. Pediatrics 2000;105:1345-8.

7 Jaspan T, Griffiths PD, McConachie NS, et al. Neuroimaging for nonaccidental head injury in childhood: a proposed protocol. Clin Radiol 2003;58:44-53.

8 Cohen RA, Kaufman RA, Myers PA, et al. Cranial computed tomography in the abused child with head injury. Am J Roentgenol 1986;146:97-102.

9 Zimmerman RA, Bilaniuk LT, Bruce D, et al. Computed tomography of craniocerebral injury in the abused child. Radiology 1979;130:687-90

10 Ewing-Cobbs L, Prasad M, Kramer L, et al. Acute neuroradiologic findings in young children with inflicted or noninflicted traumatic brain injury. Childs Nerv Syst 2000;16:23-33.

11 Rao P, Carty H, Pierce A. The acute reversal sign: comparison of medical and non-accidental injury patients. Clin Radiol 1999;54:495-501.

12 Alexander RC, Schor DP, Smith WL Jr. Magnetic resonance imaging of intracrahial injuries from child abuse. J Pediatr 1986;109:975-9.

13 Barlow KM, Gibson RJ, McPhillips M, et al. Magnetic resonance imaging in acute non-accidental head injury. Acta Paediatr 1999;88:734-40.
14 Sato Y, Yuh WT, Smith WL, et al. Head injury in child abuse: evaluation with MR imaging. Radiology 1989;173:653-7.

15 Haseler LJ, Arcinue E, Danielsen ER, et al. Evidence from proton magnetic resonance spectroscopy for a metabolic cascade of neuronal damage in shaken baby syndrome. Pediatrics 1997;99:4-14.

16 Biousse V, Suh DY, Newman NJ, et al. Diffusion-weighted magnetic resonance imaging in shaken baby syndrome. Am J Ophthalmol 2002;133:249-55.

17 Suh DY, Davies PC, Hopkins KL, et al. Nonaccidental pediatric head injury: diffusion-weighted imaging findings. Neurosurgery 2001:49:309-20.

18 Johnson DL, Boal D, Baule R. Role of apnea in nonaccidental head injury. Pediatr Neurosurg 1995;23:305-10.

19 Atlas SW. MR imaging is highly sensitive for acute subarachnoid haemorrhage...not! Radiology 1993;186:319-23.

20 Geddes JF, Vowels GH, Hackshaw AK, et al. Neuropathology of inflicted head injury in children. II. Microscopic brain injury in infants. Brain 2001;124:1299-306.

21 Geddes JF, Hackshaw AK, Vowels GH, et al. Neuropathology of inflicted head injury in children. 1. Patterns of brain damage. Brain $2001 ; 124: 1290-8$.

22 Morris MW, Smith S, Cressman J, et al. Evaluation of infants with subdural hematoma who lack external evidence of abuse. Pediatrics 2000;105(3 pt 1):549-53.

\section{Chronic non-bacterial osteomyelitis}

hronic non-bacterial osteomyelitis (CNO) in children may present as single or multiple lesions and there may be a single episode or recurrent episodes. Chronic recurrent multifocal osteomyelitis (CRMO) is thought to be the childhood equivalent of the synovitis, acne, pustulosis, hyperostosis, and osteitis/osteomyelitis (SAPHO) syndrome that affects adults. Although there have been reports of bacterial growths from CRMO lesions (Staphylococcus aureus, Mycoplasma hominis, Propionibacterium acnes, Bartonela henselae, and Coxiella burnetti have all been reported) most microbiological studies, including PCR testing, have given negative results. Antibiotic treatment does not work but anti-inflammatory agents do (non-steroidal anti-inflammatory drugs are effective and steroids, interferon $\alpha$, interferon $\gamma$, biphosphonates, and tumour necrosis factor antagonists have been used). Little has been written about the long term course of the disease but a follow up study has recently been reported from Germany (H J Girschick and colleagues. Annals of Rheumatic Diseases 2005;64:279-85).

They followed up 30 children (aged 2-17 years at symptom onset, mean 10.3 years; 21 girls) for at least 5 years (mean 5.6 years). The mean age at diagnosis was 11 years (range 221 years) and time from first symptoms to diagnosis 5-9 months. Nine children had a single lesion and no recurrence, three had a single lesion on more than one occasion, nine had multiple lesions with no recurrence, and nine had multiple recurrent lesions (CRMO). The most commonly affected bones were the clavicle and the calcaneum but altogether 59 different bones were affected. Only one patient had multiple lesions in any one bone. The clinical features were those of osteomyelitis. Twenty-four children had joint involvement; seven had cutaneous pustules.

Laboratory test results (haemoglobin, leucocyte count, erythrocyte sedimentation rate (ESR), serum ferritin, serum IgG) were generally normal. Serum IgM was moderately raised in four multifocal cases and serum IgA in three. Tests for antinuclear antibody were negative. Twenty-five children had biopsies of bone lesions and all showed the histology of chronic osteomyelitis usually with reparative changes (marrow fibrosis, trabecular osteoid apposition, and periosteal hyperostosis). Patients with CRMO were more likely to have granulocyte infiltration and hyperostosis. Microbiological investigations were negative.

Twenty-seven patients were treated with naproxen, which appeared to be effective in general. Children with a single episode needed less lengthy treatment than those with recurrent episodes (mean 13.4 months vs 25 months). One patient with non-relapsing multifocal disease and four with CRMO were treated with prednisone in addition to naproxen before achieving remission. Other treatments (sulfasalazine, biphosphate, hyperbaric oxygen, surgical decortication) were apparently ineffective. All lesions were quiescent 5 years after disease onset. Bone growth was not affected but one patient had difficulty eating because of unilateral mandibular hypertrophy.

There appears to be a spectrum of $\mathrm{CNO}$ ranging from a single non-recurrent lesion to recurrent multifocal lesions (CRMO). The cause is not known but the prognosis is good. 